\title{
Scientific writing and publishing with IMRaD
}

\author{
Marius Teodosiu's
}

\section{Why scientific publishing?}

\section{"Publish or perish"}

The current orientation towards grading scientists by the amount and quality of published articles (quantified by different indicators number of citations, the Hirsch index or the
Teodosiu M.. Scientific writing and publishing with IMRaD. Ann. For. Res. 62(2): 201-214.

Abstract. The current trend of grading scientists depending on the amount and quality of scientific papers led to a pressure in publishing, which now tends to be reduced by opting for high-quality publications. Experimental research, the largest part of scientific research, is by far best presented in research articles - abbreviated IMRaD - a form of presentation developed in the last 100 years and now almost standardized. Even though writing seems simple to ever scientist, its rules can be learned by practice. It is of utmost importance that a well conducted science is presented in a properly written manuscript, as there are cases when one was delayed in publication due to a bad scientific writing. As with any other type of research papers, IMRaD also requires good knowledge when its main parts are concerned, in structuring its different sections, in using the most suitable language, in conducting the final improvements before submitting to a journal, or in interacting with other scientists during the peer-review process. Authors can follow the basic rules suggested in this paper and have to pay attention to the ethics of scientific writing throughout the development of their manuscript. The final reward, the publication, will be the accompanied by the reader satisfaction, the number of citations and, if it is the case, the mentoring of a young colleague in writing research.

Keywords: scientific publishing, publish or perish, scientometrics, documentation

Authors. "Marin Drăcea" National Research-Developemnt Institute in Forestry (INCDS), Station Câmpulung Moldovenesc, Calea Bucovinei 73b, 721500 Câmpulung Moldovenesc, Suceava, Romania.

§Corresponding author: Marius Teodosiu (marius.teodosiu@gmail.com)

Manuscript received November 27, 2019; revised December 20, 2019; accepted December 28, 2019; online first December 31, 2019. 
a "race to publish", which could sometimes generate "forced publications" of poor science (Sanal 2006, Carneiro et al. 2007).

The number of scientific journals, the modern tools easily producing manuscripts (online documentation, word processors), the knowledge provided by computers (data analysis), or the number of scientists also contribute to this fact matter (Hartemink 1999). Getting a job or a research grant, a promotion or advancement career-wise, professional recognition, are all related to the quantity and quality of the personal published science (Miller et al 2011, Kampourakis 2016), although this often depends on opportunity, funds, collaborations etc., as well (Sanal 2006).

Being so focused on promotion - salary grants, some authors have considered the publications a currency in finding jobs and funding (Strange 2008) or more of a business than science (e.g. dissemination, the final act of research, Sanal 2006). In this race for being published the question of how much time is left for reading arises, as cutting-edge science and no awareness of it cannot be accommodated together (Hartemink 1999). In extraordinary situations, chasing publishing and accumulating articles has been associated by others with a form of obsessive-compulsory (OC) disorder of the scientist (Lippi 2017).

\section{If not publishing ...}

On the other hand, some scientists prefer to conduct scientific experiments instead of writing up (Belt et al. 2011) or even to return in the field, cases in which one can be accused of wasting the time of collaborators and misusing money possibly much-needed for another research (Clapham 2005). The argument sustaining these beliefs is that the final acts of every research are the publication and dissemination of results to the larger public (Day 1998). When this does not happen, the whole effort behind the research has no value (Link 2015), because publication is an obligation per se for scientists, and does not include academic sat202 isfaction (Clapham 2005). The most straightforward statement about scientific publishing is probably that of Gerard Piel": "Without publishing, the science is dead" (Day 1998, p. 11).

\section{Another approach?}

Sometimes, new ideas and concepts - the very essence of science - are lost in the huge number of papers, with small variations among topics. Moreover, if a paper has a large authorship, to identify the importance of individual intellect in producing science is almost impossible (Sanal 2006). Science and its final act, publication, is, in fact, the fate of ideas (Miller et al. 2011), expert feedback and their independent authentication by peer-review (Cargill and O'Connor 2009).

The history of science contains enough examples perceiving indifferently instancces of future seminal research: that of Gregor Mendel, the penicillin of Alexander Fleming (Nobel prize, 1945) or the DNA double helix of Watson \& Crick (1953) (Katchburian 2008). Or, mentoring the process of learning the scientific writing, as young researchers write, while the senior researchers just do revise (Shokeir 2014). In response to these aims sustainable acquiring of information, writing to learn, writing for others and to share knowledge - some re-consider the grading of scientists by high-quality publications as an alternative which could reduce the pressure of publishing (Miller et al 2011).

To support this: “... I believe that what matters more is not where you publish, but what you publish."3

\footnotetext{
${ }^{2} \mathrm{He}$ was a long time Editor-In-Chief of the important journal Scientific American (from 1948), a promotor of science, distinguished with many prizes, such as the e UNESCO Kalinga Prize for the Popularization of Science (1962).

${ }^{3}$ Prof. Oliver Smithies, Nobel Prize for Psychology in 2007. Web: https://www.lindau-nobel.org/oliver-smithiesthe-origin-of-ideas/. Accessed 11.2019.
} 


\section{IMRaD}

Among the many types of scientific papers research articles and research notes, review articles, editorials, comments, essays - the experimental research is properly presented in the form of research articles, abbreviated IMRaD, standing for the main sections: Introduction, Material and methods, $\underline{\text { Results }}$ and $\underline{\text { Discussion. }}$ Horton (1995) found similarities between this four-element structure with the Aristotle's "Rhetoric" (1 - introduction, 2 - narration, 3 - proof, 4 - epilogue) (Jenicek 2006). The first use of a structured presentation for a research seems to be attributed to Pasteur, in his "Etudes sur la Biere" (1876, France), while Philosophical Transactions of the Royal Society of London (1665, England) is considered the first scientific journal (Day 1998).

This way of presenting research has been observed and experimented with in the past 100 years (ICMJE 2017, p. 102; Day 1998, p. 9). The final form of a research article was adopted as standard by the National American Standards Institute in 1979 (Day 1998, p. 12). Depending on the format and the editorial policy of a journal, there could be many variants: AIMRaD, AIRDaM (Science), AIM(RaD)C (where A is the abstract), or even forms not including all the parts.

The aim of the paper is to advocate the scientific publishing and how to write the different parts of a research article. Sometimes, the proper manner of writing is neglected and this can affect the publication of the manuscript: even good science could be delayed in publication due to a unsuitable way of writing (Day 1998, p. 4). For this purpose, the common flow of documentation and subject finding, choosing the journal or revisiting the manuscript will be presented after. I will refer to the exhaustive IMRaD as including all its components, and for each will be giving suggestions for writing, structuring and improving, in order to be submitted and revised by a journal.

\section{Writing the "extended" IMRaD}

\section{The title}

Choosing a proper title is of great importance, as it represents the first information a potential reader encounters and a reason to continue with the summary and the whole paper. What does a good title look like? Clear, concise and honest regarding the content of the paper. "Less is more", the short titles being preferred to the long ones. To find a good title, it is better to devise options and to improve them during the writing of the paper; the final form of the title should be chosen after the manuscript is finished (Gemayel (2016).

Frequent errors. The numbered titles, proposing a series of articles ("How to write ...: I. Title") or the general concepts, starting with "Research ...", "Analysis ...", "Studies ..." or "On ..." are not recommended. Those including ":" followed by explanations or conclusions tend to switch the reader's attention on the previous part ":.". Other authors advice avoiding affirmative titles, named "assertive sentence titles" (Rosner 1990).

\section{Authors and authorship}

One of the main debates concerning the ethics in publishing is related to the question: "Who can be author of a scientific paper?" From the numerous rules, The International Committee of Medical Journal Editors (ICMJE 2017) provide a complete document (well known in the past as the Vancouver Protocol, since 1978). According to this, an author should simultaneously fulfill the rules found in Table 1 .

When not all the conditions are met, different people contributing to the manuscript can be mentioned in the "Acknowledgments" section: technical staff collecting the data, people involved in the design of research, in the interpretation of data or in language proof (Jones 2003).

To overcome the authorship problems, in 
Table 1 Authorship rules (ICMJE 2017)

\begin{tabular}{ll}
\hline 1 & Substantial contribution to research design [OR] analysis and interpretation of data \\
\hline $2[\mathrm{AND}]$ & Writing [OR] critical revision of manuscript \\
\hline $3[\mathrm{AND}]$ & Final approval of the version submitted to publisher \\
\hline $4[\mathrm{AND}]$ & $\begin{array}{l}\text { Approval for all the contents of the manuscript, including the precision and integrity of data } \\
\text { and results }\end{array}$ \\
\hline
\end{tabular}

some journals the contribution of each author to the manuscript has to be explicitly specified: A.B. has the research idea, C.D. and E.F sampled the data, A.B. and G.H. performed the statistical analysis, A.B. with the contribution of C.D. and G.H. have written the manuscript, and all the authors analyzed and discussed the results, revised and approved the manuscript.

\section{Number of authors}

An article can have one to tens/hundreds/thousands authors ${ }^{4}$. The first known article of the latter is "100 Autoren gegen Einstein" (100 authors against Einstein), which formulated criticism against Einstein, including on the theory of relativity. Genomics and physics are two other fields where large co-authorship is frequent: Leung et al. (2015) - 1014 authors, with 900+ students (in genomics), and Aad et al. (2015) - 5154 authors, research of CERN teams, in physics. Another article with a large authorship, in order to impress by adhesion to it, was Ripple et al. (2017) - "World Scientists' Warning to Humanity: A Second Notice", with 15372 authors from 184 countries.

\section{Order of the authors}

The main (first) author is the one who has the most significant contribution to the paper, although this position can sometimes be attributed to a young researcher, for encouragement and confidence boost (Carneiro et al. 2007). The first author is also responsible for the order of the co-authors, which depends on their contribution to the paper (Volmer 2007).

\footnotetext{
${ }^{4}$ Due to brevity, these references will be shorten to the first and last authors.

In the case of a paper with many authors, e.g. resulting from a large international collaboration (a research project, a research network), the first author(s) has main contributions to the article, then being listed „middle" co-authors in alphabetical order, while the last one is the project leader. When the manuscript is part of an ongoing $\mathrm{PhD}$ thesis, the first author is the PhD student, while the last one is the coordinating professor.

The corresponding author is the one responsible for communication related to the paper: with the journal's editorial team and the co-authors during the publishing process, and also after publication, with scientists interested in details of their article.

\section{Abstract and keywords}

The abstract reproduces, in short, the general structure of the article. It has an average of 200-300 words (with limits between 100-700 words) (Shokeir 2014), does not include references or abbreviations still unexplained in the text.

It does not take into account the Discussion section (why it is shortened IMRaC) and it contains the following parts: (i) the introductory sentence, an overview of the paper and its aim, (ii) the methods, (iii) the most important results and (iv) the conclusions, which should be strictly based on individual results (Cargill \& O'Connor 2009, p. 73). As the manuscript requires many improvements of the contents until the final form (Branson 2004, Shah 2017), it is recommended to write the abstract last, when all concerns regarding the paper have been clarified.

Usually, there are 3-6 keywords, different 
from the words in the title; this could improve the relevance of the paper when it is searched in databases of literature. Some of these databases even offer supporting information in form of controlled vocabulary (National Library of Medicine's - USA, Medical Subject Headings - MeSH) (Fernandez-Llimos et al. 2017).

For the same indexing reasons, apart from the above types of abstracts, called unstructured, where the content is presented in one single paragraph (Pearce \& Ferguson 2017), there are structured abstracts, which follow the main sections of the article, as well, but explicitly marked with titles and in distinct paragraphs, which can be further useful in reviewing the literature using modern tools based on natural language processing (Marshall \& Wallace 2019).

\section{Introduction}

This part of the paper is a short review of knowledge, which presents the gaps in knowledge and anticipates the main subjects of the paper. Usually, it is structured in 4-6 paragraphs (for brevity, 4 in hereafter examples).

The general framework related to the subject to be analyzed is presented in the first paragraph (P1), followed by the main aspects already presented in literature, along with the main reached conclusions (P2). The gaps in knowledge uncovered or not enough deepened could be emphasized in the $3^{\text {rd }}$ paragraph (P3), giving the chance for the research questions of the article to be anticipated. While in P1 it is usual to cite primary sources, $\mathrm{P} 2-3$ require recent literature, in order to prove a general understanding of the topics by authors, and key questions which the authors could try to answer. Since this part is a place where abbreviations could appear first, they should remain the same throughout the paper. The last paragraph (P4) presents the aim and purpose of the paper, respectively the main research questions (among 1-3). When choosing the subject of the paper, a narrow topic is preferable, where a contribution can be brought, respectively one on which the authors have enough experience. Otherwise, a serious time investment is necessary, in order to get the newest approaches of the topic. At the end of the research questions, a paragraph with an additional argumentation on the aim, hypotheses and research questions or the main expectations of the paper (with citations, if the case) could be included. For congruence among the research questions and their answers (as obtained from different section of the paper, e.g. Results, Discussion), these may be adjusted and reformulated at the end of the work (Belt et al. 2011).

Frequent errors. Two of the most common mistakes found in this section are linked to length and originality. The first one consists in long introductions which, at the same time, tend to be too general and vague. In regard to originality, this is the section where authors have a tendency to plagiarism, sometimes creating a so-called "mosaic" introduction, unfairly taking credit for others' ideas.

\section{Material and methods}

If this section were to be resumed through keywords, those are "reproducibility"and "cooking book" (Day 1998). This section should be written concisely, but with sufficient details so that anyone interested in the topics of the paper can replicate the research. The structure of this section can vary, and these parts could be: (i) site location, (ii) materials, methods and laboratory analyses and (iii) statistical processing of the data.

(i) Site location defines the sampling locations: name, coordinates, (a)biotic characteristics (e.g. altitude, climate); this can be done in a table or as a map (but without redundancies in information).

(ii) The methods and the materials must be described intricately enough to reproduce the proposed experiment. In case of different protocols or laboratory instruments, all details 
will be given (e.g. the complete name of the protocol, device, producer, country of provenance).

(iii) Statistical data analysis will use the most adequate methods, according to the proposed aim. During the documentation stage, the sections dedicated to the methods could give hints on a better data processing. The advanced statistical methods are not a purpose alone, but they could often draw more information from the data, compared to the classical statistical approaches. For these, a brief presentation is enough, but in a situation of methods less present in literature, a more detailed description is recommended.

The software applications (including libraries, packages) are indicated at the end of this section. For the same reason of reproducibility, some authors also offer the code associated with the programming of the statistical application (mostly as additional material).

\section{Results}

The Results section is "the heart of the paper" (Kallestinova 2011) and requires an organized presentation, as almost always more information than can be included in the paper results from data processing (Sharp 2002). The two tasks that must be simultaneously accomplished are the data presentation (as tables, figures, text), and the analysis of these results (Shokeir 2014).

\section{Data presentation}

Presentation in tables contributes to concision and offers the possibility of detailed information, which can be further compared in rows and columns. The tables (and figures) must be self-explanatory: the reader should understand the whole table without searching through the paper for abbreviations. The additional information must be provided as a note, as well as the details of the table head (ICMJE 2017, p. 106). Both tables and figures will be included in the text after their citation.

In figures, more general data can be presented (e.g. trends), without overlapping with data in tables. The figures should be clear, without many elements, in a recommended proportion of 3:2 and with a common formatting across the figures (letters, numbers, and symbols). When there are few elements in a figure, it can have also a frame, otherwise, it is not suitable. In case of figures from other sources (publications), this must be specified in the title of the figure and the author(s) must grant the written acceptance of the publisher, in order to use it.

The order of presentation should follow the same topics from Introduction and Material and methods (as it applies) and must include the relevant results only (e.g. about 4 tables and figures, respectively), while the rest can be sent as supplementary material. Some journals can require the original data to be posted online as additional material.

\section{Analysis of the results}

Across the Results section, the analysis will be concentrated on the key outcomes (Belt et al. 2011). Just a presentation of the results will be included here, without any interpretation related to other authors, which is a matter of Discussion. The ideas transmitted to the reader will be designed around the most important tables and figures. Depending on context, the tables can include sample size, summary statistics (e.g. average, standard deviation), degree of freedom, $p$-values (Drotar 2009). When figures are concerned, their comment will begin with a conclusion and be followed by the technical details contained (Gemayel 2016). The measurement units will be in International System (SI), metric system (e.g. Celsius for temperatures), while in the case of composed, it is preferred to not use the fraction $\left(\mathrm{m}^{3} / \mathrm{ha}->\right.$ $\left.\mathrm{m}^{3} \mathrm{ha}^{-1}, \mathrm{~m} / \mathrm{s}->\mathrm{m} \mathrm{s}^{-1}\right)$.

Frequent errors. This section it is not the place to present used methods or to compare the obtained results with findings of others: 
this is a purpose of other specific sections. When two of the sections are submitted together ("Results and discussion"), the comparison and discussion of other papers can be hidden in the large mass of results, which could be understood as a weak knowledge of the literature, as well. Another issue can be the redundant presentation of the same data in tables and figures, or the missing of the tables and figures citation in the text (including those from the supplementary material).

\section{Discussion}

This section it is one of the most difficult to be written and can sometimes be the weakest element of a paper (Perneger 2004, Jenicek 2006). This is the place where the competencies and the skills of the author can be observed: in a critical analysis of their own results, or in the performance of the study, compared to similar research.

When beginning to write the discussion part, it is recommended to have in mind the importance of the study, its relatedness with similar research, its limitations and implications (Hess 2004). Some authors advice devising a five parts structure of this section, containing: (i) a short introduction, (ii) interpretation of results, (iii) limitations and their implications, (iv) recommendation for the practice, (v) perspectives, future research and (vi) conclusions, if this will be not a separate section (Shokeir 2014, Drotar 2009).

(i) A short introduction. When starting to write this short introduction it is useful to re-read the research questions to be answered (Branson 2004). In about three phrases (Shidham et al 2012), the new findings and their possible explanations could be summarized (ICMJE 2017, p. 105). The order of presentation should follow the order of the similar ideas in Results.

(ii) Interpretation of results. In this second part, in few paragraphs, the obtained results will be set side by side with already pub- lished similar research (Shidham et al 2012). For an overall perspective, some primary (historical) references can be employed, while the newest/relevant papers will serve for interpreting the results (e.g. the references from the $2^{\text {nd }}$ paragraph of Introduction). This is the critical part of Discussion, where the causes and hypotheses to support the identified similarities and differences in results will be analyzed. In this part tables and figures are not allowed, but they can be referenced to in the section Results.

(iii) Limitations and their implications. It is highly recommended that the author(s) honestly discusses the limitations of the study, instead of them being observed by a referee or even readers (Hess 2004). Moreover, what is missing could offer new ideas and research opportunities for people interested in the same subject (Drotar 2009).

(iv) Recommendations for the practice. This part could include findings for implementing in practice. Some journals explicitly request a paragraph/sub-chapter of practical ideas.

(v) Perspectives, future research. This part is somewhat programmatic and related to future research. The perspectives and future directions of research will be based on the results, experience, and the new ideas emerging in part (iii) could be developed here.

(vi) Conclusions. If the authors do not intend to have a separate chapter of conclusions, the last paragraph from discussion could be the right place (suggestions, bellow).

\section{The writing approach}

The writing approach will have in mind both a specialized and a generalist audience (Gemayel 2016). The formulations should be done with precautions, but within limits, while the conclusions should be based on one's own results (Docherty \& Smith 1999). At the end of the exposes, the verbs will reflect these precautions ("seems ...", "suggest ...") (Lippi 2017). 
There are also suggestions on how to begin a sentence: "In this study ...", "Our results indicate ...", "In comparison with the results obtained by ..." (Kallestinova 2011). For a better readability, the parts (i-vi) could have titles and can be structured with sub-chapters.

Frequent errors. One of the frequent errors is the same tendency to plagiarism and the confusion with the style of writing the Introduction (from general to particular - the aim of the paper), while in the Discussion the results are within the context (Kallestinova 2011). It is a bad practice to present results and conclusions not supported by research of one's own. Regarding the style of writing, speculations, superlatives and the lack of modesty about results are not encouraged.

\section{Conclusions}

The conclusions should concentrate, in a clear and simple language, the personal findings resulted from the paper research in about 4-6 sentences. Additionally, at the end, the possible future directions of research could be mentioned.

\section{Acknowledgments}

This is an optional section, and is a sign of gratitude from the author(s) to institutions or people having facilitated in any way the research: supporting institutions/projects (with names, acronyms), people who cannot be considered authors (the people contributing to field data collection, to some statistical analysis, to proof-read), anonymous reviewers contributing to the improvement of the manuscript. The writing style should be concise and without commendatory formulations.

\section{References}

In this section are listed all the papers cited across the manuscript, with a perfect accordance between cited works and references. In the text, it is not necessary to cite more than 3-4 published works (articles, books or works having available a DOI - Digital Object Identifier). Among these, recent articles are the best, while books are seen as of secondary importance (Shokeir 2014). Literature not available to a large audience (nor by the library exchanges) cannot be cited: works under printing or unpublished, gray literature (technical reports).

One special attention is directed to the formatting of the references according to the journal requirements (as citations in text or as full reference): this is a sign that the author(s) follow the journal requests and give attention to all the necessary details of the manuscript. Dozen of formatting styles are available for the used reference managers, sometimes on the websites of said journals. If available, including DOI on a reference is a common practice.

\section{Supporting Information}

Under different names (e.g. Appendix, Supplementary Material), this section is necessary especially in the case of an exhaustive study and it suggests a preoccupation of the authors for concision. Here could be included processed information of second order importance from Results (tables, figures), primary data or software (code, package) used in statistical analysis. In the manuscript, these are mentioned after the references and it is usual for them to be available only in the online versions of the papers.

\section{Other information}

Depending on the requirements of each journal, other information may be required, e.g. conflicts of interest of the authors, contributions to authorship, ethics declarations (use of the subjects in experiments, environmental protection regulations). 


\section{The manuscript, from documentation to submission}

Apart from the structure and requirements of a research article, there are some other issues to consider when writing a manuscript: documentation and formulation of the research questions, order of writing the manuscript sections, its improvement or how to choose a journal and to formulate a cover letter (Cargill \& O’Connor 2009, p. 81).

\section{Documentation. Electronic management of literature}

In the era of digital documents and the Internet, a search engine is probably the first source of obtaining information on a possible topic of interest. Therefore, the existence of a "subdivision" related to scientific papers is not surprising, it being called Google Scholar and probably the largest database with scientific documents. Other alternatives are Web of Science (Clarivate Analytics) - providing references about the ranks of the scientific journals, Scopus (Elsevier), another alternative of the last and, intended (not necessary only) for the field of medicine, PubMed - US National Library of Medicine. Some of these also grant different scientometric indicators to rank the scientific performance (not only of a journal), but of a scientist or even of a scientific paper.

A first search in database for a topic could provide hundreds of records; from these, the most valuable are the review articles, which concentrate the essential related knowledge, and the research articles (the primary papers, the newest) along the "hidden" literature - their references.

Most of the time, the websites of the publishers and some databases have the possibility of "click \& save" the information regarding a scientific paper (including the paper, too) in some reference managers ${ }^{5}$, which interact with the

${ }^{5}$ More details on Web: https://en.wikipedia.org/wiki/ Comparison_of_reference_management_software. Accessed: $11 . \overline{2} 01 \overline{9}$. text processors and allow an easy citation and particular styles of formatting the references, together with their automatic management.

As personal expectations from a good reference manager, I would mention: to be free, available on many operating systems, to work both online and offline, to offer keywords classifications (from title or even contents), to click \& save data by working with the major browsers (Firefox, Chrome), to be integrated with the text processors of different operating systems (including the most important free distributions), or to have the possibility to automatically extract the highlighted text from the associated .pdf file of a record from databases. Another option present in most the reference managers is the opening of a collection with hierarchical levels, similar to the paper structure.

\section{Order of writing the sections}

In addition to the above recommendations regarding the writing of IMRaD sections, different authors propose different starting points: to write just the abstract and the title when ending the manuscript (Day 1998, p. 23) or, more detailed, different successions: methods-results-discussion-introduction_reference-abstract-title (Shidham et al 2012), methods-results-introduction-discussion (Kallestinova 2011), (introduction)-methods-results-discussion-introduction-conclusions_abstract-title (Belt et al. 2011). The last example appears to be very practical: writing a preliminary part of the Introduction ensures a clearer view of the gaps in knowledge and the aim of the paper. This will be then polished when ending the manuscript.

\section{Revisions and reformulations}

Writing is not an easy task, therefore some authors (Kallestinova 2011, Shokeir 2014) might surprise authors when saying that at least three revised versions for a pre-submitted manu- 
script are something common (please see bellow). As encouragement, others evoke the ending of "A farewell to arms", rewritten 47 times by Ernest Hemingway (Rubin et al. 2017).

Version 1: the maximum amount of information. The first draft of the manuscript is responsible for the "ideas" on the subject, based on a first literature reading and data processing, with all the information presented in a unpolished form. It is the case of Introduction and of other parts too and it is expected that the author(s) don't synchronized different information across sections, as suggested before.

Version 2: ordering and revision of ideas, tables, figures. This second verification is the one which prepares the manuscript for its final form; in this phase, a rhythm of correcting 4 pages/day is suggested (Kallestinova 2011). The whole text will be verified while keeping in mind the style of scientific writing - impersonal and descriptive, and its requirements - simplicity, clarity and conciseness.

For a better readability, the text will be separated in paragraphs, with the first sentence announcing the contents, and the last, being conclusive. The advancing between paragraphs will be checked for continuity, using link words ("still", "however", "also", "beside") (Day \& Gastel 2006). The component sentences will be clear, not too long (1-2 lines) and with an identifiable subject (Gemayel 2016, Lippi 2017). Verbs tenses will be used according to the section: present in Introduction, Discussion and Conclusions, and past in Material and methods and Results (Shokeir 2014). The above mentioned requirements of the tables and figures will be checked once again.

Version 3: separating the issues of secondary importance. For the last check, it is suggested that the corrections are done on a printed version of the manuscript (font size 14 points) (Kallestinova 2011).

\section{Which journal?}

Due to some particularities of each journal 210 (the structure of published papers, formatting, scientific ranking), it is advisable to choose the journal to which one submits the manuscript before starting to write it. Lippi (2017) suggests a set of four criteria: (i) coincidence among the aim of the paper and the subjects published by the journal, (ii) scientific level of own research, (iii) the impact factor of the journal and (v) publishing facilities (costs, open source, publication speed). Establishing the target journal should be a compromise between dreaming (the best journal) and realism (the journal with a paper of similar quality, e.g. from the references).

Some authors choose from the beginning a submission in two (or more) steps, with the first being to a highly ranked journal, and further until published. Besides this, some publishing houses, with many scientific journals, provide different tools (electronic forms) to undecided authors, in order to aid them in choosing from a list of the possible journals, according to the paper content (e.g. based on the abstract).

An extended list of journal requirements related to the submitted manuscripts is available on its Website, usually in a section dedicated to the authors (e.g. "Authors Guidelines"), which presents information concerning the contents or the formatting rules for a manuscript. These are not always followed by authors, therefore the editors can be dissatisfied and even reject the manuscript (Belt et al. 2011). Regarding articles in English, the proof reading should be done by a native speaker, who is familiar with the technical language used.

\section{Formatting the manuscript}

In order to facilitate the peer-review, the manuscripts of research articles have a standard formatting style: 1.5-2 line spacing with line numbering, no paragraph indentation, common font (Arial, Times New Roman), spacious enough page margins $(2-3 \mathrm{~cm})$. Submitting a manuscript formatted in this respect denotes care to the editor/referee, translated in the way of conducting the research, too (Lippi 2017). 
The final manuscript will include: (i) Title page no. 1, (ii) Title page no. 2, (iii) Contents, (iv) Supporting Information (which can be attached as separate documents).

(i) The title page no. 1 separates the content of the manuscript from the authors. It contains: the title, the keyword, the authors and the number of words (Shokeir 2014), and can include a shortened version of the title (the running title). If the authors are concerned, the first name, last name and a number corresponding to the affiliation (institution and contact - address, phone, fax, email) will be indicated. To indicate the corresponding author, a different symbol will be used. (ii) The title page no. 2 includes only the title of the manuscript; this, together with the further contents, will be sent to reviewers. (iii) In the contents of the paper, the emphasis is on the story (the text), therefore the tables and the figures are inserted at the end, in two separate sections, even though their position in text will be marked.

As it is available to many of the word text processors, the use of headings is recommended, for an easy navigation and editing of the manuscript.

\section{The cover letter}

The cover letter is the document which attempts to motivate the editor to accept the manuscript for peer-review/publication. It should be clear, honest and let know the editor about the importance of the manuscript's publishing in their journal (Cargill \& O'Connor 2019 , p. 83). The cover letter may include: the title of the manuscript, the authors (and affiliations), a short description of the paper, the importance of the paper and a declaration of conformity (e.g. there is no conflict of interests, no plagiarism, and the paper is acquired by all the authors).

\section{The editorial process}

\section{Editors and confidentiality}

A manuscript, to be attractive for the editor of a journal receiving hundreds or thousands of such papers a year, should have some characteristics: topic novelty (e.g. by using the most recent/significant literature), a proper experimental design and data processing and analysis, and to be well written, e.g. to follow the above mentioned common rules of writing the IMRaD. The editor, as manager of the journal, takes responsability for the decision of publishing.

On the other hand, according to the international publishing regulations (ICMJE 2017, p. 93), the authors are confident about the confidentiality of their identity or the content of the manuscripts, which will not be used by the editors or reviewers in any way. Once the paper is transmitted to the journal (more often through a specialized electronic platform), the corresponding author should wait for the decision of the editor.

\section{The peer-review process}

Any revision of a manuscript is a good opportunity to improve the manuscript, which is done on the basis of the voluntary and time consuming effort of the reviewers. This means generosity and academic services, because they contribute to the progress of anonymous works, a reason for them to be included in the section "Acknowledgments" (Shidam et al. 2012).

The reviewers and the peer-review

After the main editor or an associate editor verifies the manuscript, the paper is submitted to peer-review. Depending on the journal, there are many types of peer-review, the most frequent one being double blind (the authors and the reviewers are anonymous to each other); 
the non-anonymous and open reviews, where all the process is visible online for anyone are rarer.

To review a manuscript, the editor contacts reviewers specialized on the manuscript topics (usually, 2 reviewers not finding themselves in a conflict of interest with the authors). Typically, the peer-review implies circulation of the manuscript between author $\leftrightarrow$ editor $\leftrightarrow$ reviewer. It is desirable for the editor to share all the assessments of the reviewers between them, in order to provide a more united approach of improvement to the paper (ICMJE 2017, p. 94).

In their commentary, the reviewer is expected to be constructive and try to improve the manuscript, using an appropriate language and criticism not offending the authors (Bourne \& Korngreen 2006). Finally, the editor will ask the corresponding author to modify the manuscript according to the reviewer's requests.

The answer of the corresponding author

After any phase of the review cycle, the corresponding author answers the reviewers' requests in a letter of response, its first phrase starting with thanking for the effort of revision. To properly reply to this, every remark must be read carefully and each will receive an answer in a polite manner. When an author does not agree with the suggestions of the reviewer, their own arguments will be exposed in a court way and, if necessary, together with citations/ references.

The author $\leftrightarrow$ editor $\leftrightarrow$ reviewer revision cycle will be run until the manuscript gets the desired level of quality. When the opinions surrounding a manuscript are totally divergent among 2 reviewers, it is usual for the editor to contact a $3^{\text {rd }}$ one to formulate a final decision.

\section{Final decision on the manuscript}

Depending on the manuscript's quality, after the first round of peer-review, the editor will communicate the conclusion to the authors: 212 the article is (i) accepted for publication, but requires minor revisions, (ii) accepted, but with major revisions, (iii) not accepted for publication (rejected). In case of poor or highly discordant manuscripts with the journal requirements, the editor can decide (iv) rejection without peer-review. Basically, a honest, well written manuscript, sent to a journal of a similar value, is accepted for peer-review.

\section{Acceptance for publication}

(i) Acceptance with minor changes applies to well written manuscripts, of quality, for which the reviewers suggested small, targeted changes. In this case, the manuscript is accepted after answering the reviewer's observations in the $1^{\text {st }}$ stage, without another intervention from the reviewers; then, the manuscript is sent for publication.

In the case of (ii) acceptance with major changes, the answer of the authors after the $1^{\text {st }}$ stage are returned to the reviewers, who decide if the authors answered all their suggestions and if it is necessary to continue with the improvement of the manuscript. A manuscript accepted with major revisions after even four stages of peer-review it is not so surprising (Kallestinova 2011).

\section{Rejection}

The rejection of a manuscript is not a pleasant event, but it can be considered an experience, useful for writing a better paper, especially in the case of young authors, where writing can be learned by practicing. And, last but not least, the authors have another manuscript, which will earn its proper place to be published (Kallestinova 2011).

(iii) If the manuscript is rejected after being reviewed, it is supposed that one/both/all the reviewers recommended its rejection and the final decision of the editor is based on this. There could be many reasons why (Shokeir 2014): irrelevance for journal, wrong methods or data processing, bad writing of the manu- 
script or unjustified conclusions.

(iv) Rejection without peer-review could have several causes (Cargill \& O'Connor 2019, p. 82): it is outside the aim and audience of the journal, the submission has no cover letter (thus is not assumed by authors), there is a big difference between the value of the manuscript and the journal expectations (by comparison with a similar journal's articles) and it uses a bad language (e.g. technical terms or poor English).

\section{A new piece of science}

After receiving acceptance for publication, the manuscript is transfered to production, so that the final electronic form of the article can be generated. This is afterwards sent to the authors for the final corrections, and they are encouraged to read it carefully and report any mistakes, because after the online publication the article can only be changed based on errata.

Depending on the editorial policy of the journal, an article is instantly associated to a future issue or, in some journals, is placed in a temporary section of the journal ("Online First", "e-First", "Latest articles"). Even here, it has the same status as an article in an issue, because by receiving DOI, its citation can be further used. In the situation of some journals which publish a large number of papers, in order to speed up the promotion of science, they post online as "Accepted articles" the very promising manuscripts in the form received from the authors (without any typesetting).

Once the paper is published online, the next step could be a small celebration, together with the co-authors, colleagues, other contributors, as a form of personal reward for a successful effort.

\section{Conclusions}

Research is an activity which implies founding, people and, finally, generosity, because the publication (its final act) should be reader-oriented, towards those who wish to receive quality science, written with simplicity, clarity and conciseness. For the experimental research, the IMRaD, in any of its forms, is a way of presentation developed after a long period of experimentation. Before publishing an IMRaD, authors should follow the basic rules suggested in this paper, the ethics in the scientific publishing and the useful interactions with other scientists during peer-review. The final gain will be the publication, the reader satisfaction and the number of citations, and if it is the case, mentoring of a young colleague in writing research.

\section{References}

Aad G. ... Woods N., 2015. Combined Measurement of the Higgs Boson Mass in pp Collisions at root $\mathrm{s}=7$ and $8 \mathrm{TeV}$ with the ATLAS and CMS Experiments. Physical Review Letters 114: 191803. DOI: 10.1103/PhysRevLett.114.191803

Belt P., Mottonen M., Harkonen J., 2011. Tips for writing scientific articles. University of Oulu, $31 \mathrm{p}$.

Bourne P.E., Korngreen A., 2006. Ten simple rules for reviewers. PLoS Computational Biology, 2(9):09730974. DOI: 10.1371/journal.pcbi.0020110

Branson R.D., 2004. Anatomy of a research paper. Respiratory Care 49: 7 .

Cargill M., O'Connor P., 2009. Writing scientific research articles: strategy and steps. Wiley-Blackwell, Chichester, UK ; Hoboken, NJ.

Carneiro M.A.A., Cangussú S.D., Fernandes G.W., 2007. Ethical abuses in the authorship of scientific papers. Revista Brasileira de Entomologia 51: 1-5. DOI: 10.1590/ S0085-56262007000100001

Clapham P., 2005. Publish or perish. AIBS Bulletin 55:390391.DOI: 10.1641/0006-3568(2005)055[0390:POP]2.0. $\mathrm{CO} ; 2$

Day R.A., 1998. How to write \& publish a scientific paper, 5th ed., Oryx Press, Phoenix, Az. DOI: 10.1590/S007402761998000300029

Day R.A., Gastel B., 2006. How to write and publish a scientific paper. Cambridge University Press.

Docherty M., Smith R., 1999. The case for structuring the discussion of scientific papers: Much the same as that for structuring abstracts. BMJ: British Medical Journal 318(7193): 1224.

Drotar D., 2009. Editorial: How to write an effective Results and Discussion for the Journal of Pediatric Psychology. Journal of Pediatric Psychology 34: 339-343. DOI: $10.1093 /$ jpepsy/jsp014 
Fernandez-Llimos F., Minguet F., Salgado T.M. 2017. New pharmacy-specific Medical Subject Headings included in the 2017 database. American Journal of Health-System Pharmacy 74(15): 1128-1129. DOI: 10.2146/ajhp170046

Garfield E., 1996. What is the primordial reference for the phrase 'publish or perish'. The Scientist 10(12): 11. DOI: $10.1136 / \mathrm{bmj} .318 .7193 .1224$

Gemayel R., 2016. How to write a scientific paper. The FEBS Journal 283: 3882-3885. DOI: 10.1111/ febs. 13918

Hartemink A.E., 1999. Publish or perish (2) How much we write. Bulletin of the International Union of Soil Sciences 96: 16-23.

Hengl T., Gould M., 2002. Rules of thumb for writing research articles. Web: https://webapps.itc.utwente.nl/ librarywww/papers/hengl_rules.pdf. Accessed 11.2019.

Hess D.R., 2004. How to write an effective discussion. Respiratory Care 49: 1238-1241.

Horton R. 1995. The rhetoric of research. BMJ 310: 985987.

ICMJE, 2017. Recommendations for the conduct, reporting, editing and publication of scholarly work in medical journals. Current Pediatrics 16: 90-106. DOI: 10.15690/vsp.v16i2.1710

Jenicek M., 2006. How to read, understand, and write 'Discussion' sections in medical articles. An exercise in critical thinking. Medical Science Monitor 12: SR28SR36.

Jones A.H., 2003. Can authorship policies help prevent scientific misconduct? What role for scientific societies? Science and Engineering Ethics 9: 243-256. DOI: 10.1007/s11948-003-0011-3

Kallestinova E.D., 2011. How to write your first research paper. The Yale Journal of Biology and Medicine 84: 181.

Kampourakis K., 2016. Publish or Perish? Science \& Education 25: 249-250. DOI: 10.1007/s11191-016-9828-4

Katchburian E., 2008. Publish or perish: a provocation. Sao Paulo Medical Journal 126: 200-203.

Leung W. ... Elgin S.C.R., 2015. Drosophila Muller F elements maintain a distinct set of genomic properties over 40 million years of evolution. G3: Genes, Genomes, Genetics g3.114.015966. DOI: 10.1534/g3.114.015966

Link J.M., 2015. Publish or perish ... but where? What is the value of impact factors? Nuclear Medicine and Biology 42: 426-427. DOI: 10.1016/j.nucmedbio.2015.01.004

Lippi G., 2017. How do I write a scientific article? A per- sonal perspective. Annals of Translational Medicine 5: 416-416. DOI: 10.21037/atm.2017.07.43

Marshall I.J., Wallace B.C. 2019. Toward systematic review automation: a practical guide to using machine learning tools in research synthesis. Systematic Reviews 8(1): 163. DOI: 10.1186/s13643-019-1074-9

Miller A.N., Taylor S.G., Bedeian A.G., 2011. Publish or perish: academic life as management faculty live it. Career Development International 16: 422-445. DOI: 10.1108/13620431111167751

Pearce P.F., Ferguson L.A., 2017. How to write abstracts for manuscripts, presentations, and grants: Maximizing information in a 30 -s sound bite world. Journal of the American Association of Nurse Practitioners 29: 452460. DOI: $10.1002 / 2327-6924.12486$

Perneger T.V., 2004. Writing a research article: advice to beginners. International Journal for Quality in Health Care 16: 191-192. DOI: 10.1093/intqhe/mzh053

Ripple W.J., Wolf C., Newsome T.M., Galetti M., Alamgir M., Crist E., Mahmoud M.I., Laurance W.F., 2017. World scientists' warning to humanity: A second notice. BioScience 67: 1026-1028. DOI: 10.1093/biosci/ bix 125

Rosner, J.L, 1990. Reflections of science as a product. Nature 345.6271: 108-108. DOI: 10.1038/345108a0

Rubin F., Maisonneuve H., Martin C., Laccourreye O., 2017. Write your first article, but ... European Annals of Otorhinolaryngology, Head and Neck Diseases 134: 371-372. DOI: 10.1016/j.anorl.2017.09.002

Sanal M., 2006. Where are we going in science? Publish and perish! Current Science-Bangalore 90: 1169.

Shah J.N., 2017. How to write abstract for a scientific journal article. Journal of Patan Academy of Health Sciences 4: 1-2. DOI: 10.3126/jpahs.v4i1.24657

Sharp D., 2002. Kipling's guide to writing a scientific paper. Croatian Medical Journal 43(3): 262-267.

Shidham V., DeMay R., Pitman M., 2012. How to write an article: Preparing a publishable manuscript! CytoJournal 9: 1. DOI: 10.4103/1742-6413.92545

Shokeir A.A., 2014. How to write a medical original article: Advice from an editor. Arab Journal of Urology 12: 71-78. DOI: 10.1016/j.aju.2013.10.006

Strange K., 2008. Authorship: why not just toss a coin? American Journal of Physiology-Cell Physiology 295: C567-C575. DOI: 10.1152/ajpcell.00208.2008

Vollmer W.M., 2007. Responsibilities of authorship. Chest 132(6): 2042-2045. DOI: 10.1378/chest.07-2051

Watson J.D., Crick F.H.C., 1953. A structure for Deoxyribose Nucleic Acid. Nature 171(4356): 737-738. 